\title{
Pedagogy and Culture: An Educational Initiative in Supporting UAE Nursing Graduates Prepare for a High-stakes Nurse Licensing Examination
}

\author{
Sharon M Brownie ${ }^{1,2,3}$, Ged Williams ${ }^{4}$, Kate Barnewall ${ }^{5}$, Suzanne Bishaw ${ }^{6}$, Jennifer L Cooper ${ }^{7}$,Walter Robb ${ }^{8}$, Neima \\ Younis $^{9} \&$ Dawn Kuzemski ${ }^{6}$ \\ ${ }^{1}$ Griffith Health, Griffith University, Gold Coast, Australia \\ ${ }^{2}$ Oxford Praxis Forum, Green Templeton College, Oxford University, Oxford, UK \\ ${ }^{3}$ Fatima College of Health Sciences, Abu Dhabi, UAE \\ ${ }^{4}$ Corporate Office, Abu Dhabi Health Services Co. (SEHA), Abu Dhabi, UA \\ ${ }^{5}$ School of Nursing \& Midwifery, Griffith University, Gold Coast, Australia \\ ${ }^{6}$ Tawam Hospital, Abu Dhabi Health Services Co. (SEHA), Al Ain, UAE \\ ${ }^{7}$ Public Health, Curtin University, WA, Australia \\ ${ }^{8}$ Population \& Social Health, Griffith University, Gold Coast, Australia \\ ${ }^{9}$ Al Ain Hospital, Abu Dhabi Health Services Co. (SEHA), Al Ain, UAE \\ Correspondence: Sharon Mary Brownie DBA, Contact Address: P.O. Box 3798, Abu Dhabi, UAE. Tel: \\ 971-501-533-508, 61-419-330-822. E-mail: s.brownie@griffith.edu.au
}

Received: December 14, 2014

Accepted: February 1, $2015 \quad$ Online Published: February 2, 2015

doi:10.5430/ijhe.v4n1p200

URL: http://dx.doi.org/10.5430/ijhe.v4n1p200

\begin{abstract}
Graduates of an Abu Dhabi transnational nursing degree struggled with the mandatory national licensing examination. Poor pass rates undermine graduate career futures and impact on the workforce capacity building contributions of the partnering transnational educational providers. This paper describes how the design and delivery of an intensive examination preparation program dramatically reversed this trend. The objectives of this educational initiative involved the design, delivery and evaluation of a program that would align with cultural learning preferences and which improve the success rates of graduates attempting the national nurse licensing examination. To achieve these objectives, the program combined a range of teaching and assessment strategies developed to reflect the specific needs of Arabic learners, build on their existing knowledge and help them engage more effectively in the learning processes required for successful performance in a high stakes examination. Analysis of data collected during program evaluation provides useful insights into the preference and experiences of nursing graduates in the UAE Emirate of Abu Dhabi. The lessons learned are applicable to Arabic learners both regionally and globally.
\end{abstract}

Keywords: Transnational nursing education, Cultural learning styles, Examination preparation, Nurse licensing, UAE

\section{Introduction}

\subsection{The context and the problem}

Historically, the UAE recruits the majority of its nursing workforce from abroad (El-Haddad, 2006; Hannawi \& Al-Salmi, 2013). A nursing workforce capacity building partnership involving Griffith University Australia and the UAE Government has been instrumental in establishing a Bachelor of Nursing (BN) program in Abu Dhabi. The partnership produces locally trained degree-qualified nurses and supports the national goal of Emiratization of the UAE health workforce (Hannawi \& Al-Salmi, 2013). The first cohort of transnational nursing degree students graduated in June 2011 and was employed by the SEHA Health Services Company, the government-owned health provider (SEHA meaning Health in Arabic). Graduates entered the 12-month graduate nurse internship (GNI) program. 
Different health systems use different methods to enable entry to practice for new nursing graduates. For example, the Australian and UK nurse professional licensing systems rely upon rigorous accreditation of educational providers as reassurance of graduate competence. Other nations such as USA, Canada and New Zealand require graduates to demonstrate competence to practice via a single nationally delivered high stakes licensure examination. The UAE has chosen to adopt the use of a single high-stakes licensure examination as the measure of competency to practice. The examination is conducted by the Health Authority of Abu Dhabi (HAAD). Success in this exam presents either a doorway or a barrier to future careers and practice of UAE-based nursing graduates. Graduates must pass this examination during their twelve months period of the GNI program.

Of the first graduating class, twenty-four of 55 graduates (43.6\%) passed the pre-registration HAAD licensing examination on their first attempt with the remainder needing to pass the examination during their GNI year. In 2012, graduates who had not passed the exam within the GNI period were suspended for 6 months at SEHA directive, but reinstated if the exam was passed during the suspension period.

The second graduating class also struggled with the exam, with only 2 of 51 graduates (4\%) passing on their first attempt. After a pattern of multiple attempts, twelve graduates remained unsuccessful at the conclusion of their induction program. In 2013, licensing requirements changed. If the exam was not passed in the GNI period, the graduate was stood down from employment as a graduate nurse in SEHA facilities. HAAD policy then prevented graduates re-sitting the exam for a 12-month period.

\subsection{Why the problem is important}

The scenario was both concerning and puzzling as the feedback from SEHA employers was that the graduates were competent beginning practitioners who despite their competence continued to fail the licensing examination. In nations with national nurse license examinations, first-time pass rates in these high-stakes examinations are a key marker of the quality of educational delivery in pre-registration programs (Pennington \& Spurlock, 2010). The struggle with the HAAD exam adversely impacted individual careers, and the reputations of the university and the in-country educational provider thereby undermining local nursing workforce capacity building aspirations.

\subsection{Developing a solution}

The need for a solution was urgent because twelve graduates who had completed their GNI year were facing permanent termination of their employment as a registered nurse because they had not passed the HAAD licensing examination.. A time period of three months was negotiated in which the employers and educational providers could identify a solution and achieve a positive outcome for these graduates.

Given this factor and the concerning overall pattern of sub-optimal performance in the licensing examination, the university partner provided additional resources to design and deliver an intensive, comprehensive review and remedial program. Program objectives of this educational initiative involved the design, delivery and evaluation of a program that would align with cultural learning preferences and which improve the success rates of graduates attempting the national nurse licensing examination - the intent being to assist graduates who had struggled with the exam, and to proactively prepare the next round of graduates for success. The program described in this paper dramatically overcame previous sub-optimal trends and delivered results commensurate with western benchmarks in national nurse licensing. To achieve these outcomes, the program combined a range of teaching and assessment strategies designed to reflect the specific needs of Arabic learners and effectively engage them in the learning process.

\section{Exploring the Literature to Inform Program Planning}

Prior to commencement, the educational team explored the literature to strengthen their understanding of 'best practices' in preparing graduates for high stakes nursing examinations; teaching students with English as a second language, examination requirements in the UAE and cultural and contextual factors relevant to candidate preparation and success. The team found that while recommendations are available on the design and delivery of review programs and remedial interventions to assist nurses preparing for high-stakes licensing exams (Crow, Handley, Shaw-Morrison, \& M., 2004; Frith, Sewel, \& Clarke, 2005; Kaufman, Baradell, Calhoon, \& Durham, 1987; Morton, 2004), most of the literature was focused on the NCLEX (Colombraro, 1989; Johnson, 2009; Norton et al., 2006; Pennington \& Spurlock, 2010). No articles were found of direct relevance to nursing within the UAE, wider Gulf Cooperation Council (GCC) States or an Arab Muslim context. Further, evaluation of existing comprehensive review programs was reported largely absent and a subject for further research (Pennington \& Spurlock, 2010).

The scarcity of published literature was not unexpected given the newness of degree-level nursing within the UAE. Thus, the authors also sought relevant grey literature, and verbal feedback from key stakeholders - all of which 
pointed to the need for consideration of locally-specific factors requiring particular attention in the program development process.

The links between culture, context, and learning were clearly highlighted within the literature, (Charlesworth, 2008; Neito, 2007), meaning it was essential that program planning considered locally unique factors (Charlesworth, 2008). The aforementioned literature highlighted a need for multi-pronged planning approach, including: a) Consultation on information such as the student group profile, current study methods, factors influencing preferred learning styles within the UAE, and exam content; b) exploration of evidenced-based guidance on the preferred learning style of Arabic students and preparation for high-stakes examination; c) analysis and procurement of available teaching and assessment resources relevant to the developing program and UAE context; d) development of a program framework; and, e) preparation of teaching and assessment materials.

\subsection{Acknowledging Cultural Learning Styles in Transnational Education}

The program was to be delivered in the Arab Muslim Emirate of Abu Dhabi, supported by an Emirati/Australian higher education partnership. A key consideration in transnational education is acknowledging differing learning preferences and styles among different cultures (Heffernan, Morrison, Basu, \& Sweeney, 2010; Sagar, 2014). Considerations of the specific program requirements are also important. Analytical and critical thinking skills are essential components of baccalaureate level nursing education with the ability to understand the nursing process and 'think like a nurse' central to success in nurse licensure examinations. Educators in the UAE are frequently challenged in moving learners away from the rote memory approaches customary for Arab learners (Cowan, 2011; Dahl, 2011). Constructivist approaches that seek to enhance learning through active interplay between knowledge, experience, and ideas are commonly used to achieve this transition (al Mahmud, 2013). However, such approaches have frequently failed to thrive among Arab learners (Dahl, 2011). A shift from rote learning to critical thinking was an essential requirement for the program to succeed in improving examination pass rates (al Mahmud, 2013; Hannah, 2006).

\subsection{Language considerations}

Language is a complex issue within the UAE health sector. While Arabic is the UAE's official language, English is the official language in the healthcare context; a safety consideration ensuring communication across a multicultural health workforce. Locally-trained Arabic speaking nurses are a valuable addition to the healthcare team, as care is delivered to many Arab speaking patients who may not speak English. The challenge facing the teaching team involved the fact that English is a second or third language for all graduates from the transnational program. All are either born in the UAE or of long-standing residence, with the majority originating from neighboring Arab speaking countries. The HAAD exam is written in English, The literature suggested that to optimize success, the program needed to be delivered in English by competent English speakers familiar with the fundamentals of English for Speakers of Other Languages (ESOL) teaching. Of importance, the program needed to ensure that learners had opportunity to interact in the target language (English) without reversion to their mother tongue to understand question content (Charlesworth, 2008; Egbert, 2005).

\subsection{Learner Confidence}

It is common for novice nurses to be "excited and scared" about their role as a graduate nurse (Burton, 2011; Dyess \& Sherman, 2009) and equally common for graduates to feel anxious and fear high-stakes licensing exams. The intended participants had a history of several previous exam failures and some had been stood down from employment. Others had also experienced significant delays between graduation and entry to the GNI program, with a resulting dip in confidence between completion of their nursing education and entry to the review program Anxiety reduction or control was therefore an essential precursor for success (Simon, McGinniss, \& Krauss, 2013).. Confidence building was noted by the authors as an essential focus within the program.

\subsection{The HAAD Licensing Exam}

The UAE has a history of higher education spanning less than forty years (Kirk, 2010), with new organizations and systems still being established. The establishment of HAAD, the introduction of its regulatory and licensing functions and the examination only occurred within the past seven years (Latham \& Watkins, 2013). As highlighted the newly formed entity has decided to implement the model of a high stakes licensure examination to confirm graduate readiness and safety for initial practice as a registered nurse. Currently, there is little information available about the three-hour 150 question multiple choice assessment, although it is anecdotally reported to be similar to the NCLEX examination and the examination of the New Zealand Nursing Council. HAAD does not provide any publicly available information regarding exam content or success rates. While a one-off opportunity was provided to 
the 2013 graduating class to attend a mock exam at HAAD premises, feedback on exam performance was not made available to students. Apart from a small number of test questions, no practice exam is available, posing a significant challenge for faculty and learners. Verbal information from HAAD officials described the exam as an assessment tool for inwardly migrating nurses with two years practice experience with the same examination used as the competency assessment tool for new graduates. While candidates receive pass/fail results following the exam, no feedback is available about strengths or weaknesses. Of necessity, program planning was based on a logically informed guess about exam content after talking with candidates who had previously sat the exam - the best source of examination information available from nurses who have passed through the examination process

The examination is offered online through an advance booking system. In support of the program, HAAD officials offered a group pencil and paper option for each program cohort, meaning participants could sit the examination immediately after the review program. Guiding literature and a sample examination was absent but it was clear to the teaching team that a different pedagogical approach was needed to meet the unique learning needs of the cohort of predominantly Arab speaking ESOL learners whose confidence was lowered following previous exam failure. Given the number of exam attempts of the initial twelve GNI graduates significant remediation was required in addition to comprehensive review of nursing knowledge. Remediation was noted as involving "the process of identifying the need to take action to remedy a situation, if unresolved, will result in unfavourable outcomes, whereas implementing intervention strategies will successfully address the situation." (Culleiton, 2009) p 26.

\subsection{Egbert's framework}

The search for effective intervention strategies provided insights relevant to teaching in different settings, particularly where English is not the first language (Atkinson, 1999; Hillege, Catterall, Beale, \& Stewart, 2014) . A framework developed by Joy Egbert, an educator known for ESOL teaching expertise and work teaching English in 'unconventional setting', asserts that efficient and effective learning can occur if teaching faculty ensure the learning environment meets certain conditions (Egbert, Hanson-Smith, \& Chao, 2007). Egbert's framework focuses on optimal learning within Computer Assisted Language Learning (CALL) environments, but has been generalized to broader ESOL environments and used to develop teaching and learning standards for English speakers of other languages. The framework involves eight conditions to optimize learning (Egbert, 2005; Egbert et al., 2007), specifically:

1. Learners have opportunity to interact socially and negotiate meaning

2. Learners interact in the target language with an authentic audience

3. Learners are involved in authentic tasks

4. Learners are exposed to and encouraged to creatively utilize the target language

5. Learners have enough time and feedback

6. Learners are guided to attend mindfully to the learning process

7. Learners work in an environment with ideal stress/anxiety levels

8. Learner autonomy is supported.

\subsection{TESOL Standards}

These eight conditions for optimal learning have been adopted by TESOL (the international professional organization for teachers of English as a second or foreign language) as an integrated system upon which standards for English teaching to non-English speakers have been built (TESOL International Association, 2002). These conditions and the TESOL standards were identified as most suited to the needs of the Arabic speaking learners and most relevant to guide development of the teaching and learning strategies for the program.

\subsection{High Stakes Examinations}

Review of literature pertaining to best practices in developing questions and preparing for high-stakes examinations (McManus \& Ludka, 2012; Twort \& Mucklow, 2011) provided additional guidance for program planning. Published research on high-stakes exam preparation programs for nurses revealed a range of effective teaching and learning strategies (Hyland, 2012; Johnson, 2009; Kaufman et al., 1987; Morton, 2004; Norton et al., 2006; Simon et al., 2013). Emerging themes were consistent with Egbert's learning requirements, including the need for learners to a) be involved in authentic tasks; b) have enough time and receive feedback; c) receive pastoral care and guidance to attend mindfully to the learning process; and, d) have opportunity to interact socially and negotiate meaning regarding their role as a nurse professional. The implication from these best practice guidelines was for the inclusion 
of activities such as three-hour practice examinations, as similar as possible to the real examination with immediate feedback through activities such as direct participant involvement in marking tests and examinations.

\section{Method: Program and Program Evaluation}

\subsection{The Program}

A 10-day comprehensive program of tutoring, learning, and examination techniques was provided for Abu Dhabi and Al Ain based graduates. This was conducted over a three-week period, after which participants sat the HAAD examination. The teaching team included both in-country and offshore academic faculty from Griffith University plus senior nurse educators from the SEHA GNI coordinator group. -the strength of partnership between service and education noted as essential to effective program planning and delivery.

The team developed teaching resources which included review material, case studies, and mock exams with consideration of optimal learning strategies to best engage learners in the learning process. On this basis, program planning used a constructivist approach to make the best use of existing knowledge and engage students in activities focused on how to determine the correct answer to questions rather than simply providing questions and answers for memorization. The use of case studies (Table 1) enabled learners to focus on using nursing process and judgment in choosing the 'best' answer for each question instead of memorizing questions and perceived 'right' answers from texts and multi-choice question banks. The program emphasized how to 'think like a nurse'. The program comprised 10 days over a three-week period. GNI candidates received 10 days of paid study leave: Nine days for instruction and one day for the HAAD exam.

Week One: Abu Dhabi candidates

- Review of medication safety and drug calculations

- Medication safety and drug calculation tests

- Review of nursing knowledge though:

- Case presentations and group discussions

○ One- and two-hour multiple choice exams

- Feedback discussions of incorrectly answered questions

- Mid-program focus groups to allow students to highlight areas of perceived weakness and request specific revision and teaching foci

- Presentations and group discussions on study skills and exam technique

Week Two: Al Ain candidates

- Repeat of Week One

Week Three: All candidates

- Review and group discussions on study skills and exam technique

- Daily three-hour mock exam (150 MCQ's) for three consecutive days

- Feedback and discussion of missed questions

- HAAD exam

The initial program acted as a pilot, involving a daily review of student results and feedback which helped guide program delivery for the following day. This program was successful, yielding an $83 \%$ pass rate (10 of 12 graduates facing career conclusion) and establishing a sound basis for delivery of the second program. The second program delivered a pattern of early passes for graduates entering their graduate induction year and established a foundation for ongoing intervention and early success for future cohorts. 
Table 1. Example case study

\begin{tabular}{|l|l|}
\hline Name & Ahmed Aljunaibi (pseudonym) \\
\hline Date of Birth & 27 Nov 1988 \\
\hline Age & 26 years old \\
\hline Gender & Male \\
\hline Height & $180 \mathrm{~cm}$ \\
\hline Weight & $82 \mathrm{~kg}$ \\
\hline Past medical history & Smokes 15 cigarettes per day for last 6 years \\
\hline Past surgical history & Inguinal hernia repair 2010 \\
\hline Allergies & Penicillin \\
\hline Current Admission & $\begin{array}{l}\text { Ahmed is BIBA (brought in by ambulance) following a MBA } \\
\text { (motor bike accident). }\end{array}$ \\
\hline Medications (pre admission) & Nil \\
\hline Social history & $\begin{array}{l}\text { Ahmed is a proud owner of a new Harley Davidson } \\
\text { motor-bike. He works in the finance department of a UAE } \\
\text { Government Department. He rides his motorbike to and from } \\
\text { work each day. His wife of 4 months, Ayesha, was notified by } \\
\text { paramedics and is on her way to hospital. }\end{array}$ \\
\hline
\end{tabular}

You are a registered nurse on a neurological/acute head injuries ward. You receive the following handover from a recovery nurse regarding. This is Ahmed Aljunaibi, (pseudonym) a 25 year old man who sustained a closed head injury this afternoon following a motor bike accident. He was taken to theatre for emergency burr hole evacuation and drainage of an subdural hematoma under a general anesthetic. The operation was uneventful and following extubation in recovery, Mr Aljunaibi was able to maintain his own airway. He is drowsy but rouseable. His GCS is 14 , with eyes opening to speech. He obeys commands and is orientated to date \& place. $\mathrm{He}$ has IV Hartmann's infusing 6/24 with further orders for N/Saline with $40 \mathrm{mmol} \mathrm{KCl}$ over 8 hours. $\mathrm{Mr}$ Aljunaibi's head is to remain elevated at $30^{\circ}$ and he is to remain NBM until a speech review. He also sustained a hairline fracture of the L) clavicle which will be treated with rest and analgesia. He has a collar and cuff insitu for stabilization. Mr Aljunaibi has an IDC (indwelling catheter) in situ.

His post-operative instructions are:

1. Monitor vital observations and neurological observations

2. Leave wound bandage intact and monitor for signs of bleeding

3. IVT and medications as charted

4. SFBC (strict fluid balance chart)

5. NBM until review

6. Notify any concerns

His observations are BP 130/82 mmHg, HR is $72 \mathrm{bpm}$, RR $16 \mathrm{bpm}$ and $\mathrm{SaO} 297 \%$ on 2L NP, Temp $36.4^{\circ} \mathrm{C}$, BGL $4.5 \mathrm{mmol} / \mathrm{L}$, GCS 14 . His pain is currently $2 / 10$ and the dressing has nil visible ooze. His neurovascular observations on his L) arm are intact. 
Questions:

1. What observations will you monitor in order to detect any deterioration in Mr Aljunaibi's condition? How often will you perform these observations?

2. Describe the main areas of assessment in the Glasgow Coma Scale and the rationale for using this neurological assessment.

3. Why is a patient suffering a head injury positioned with his head elevated at $30^{\circ}$ ?

4. If GCS score should deteriorate, what would be your immediate actions?

5. Why should Mr Aljunaibi remain NBM? What is he at risk of?

6. During admission to the Neurosurgical ward, he has a seizure. How will you maintain his safety while he is having a seizure? What risks are associated with a patient suffering a prolonged seizure?

7. Mr Aljunaibi is ordered Stat Diazepam $5 \mathrm{mg} I V$, intravenous Phenytoin $-5 \mathrm{mg} / \mathrm{kg}$ daily, IV Ceftriaxone 1 $\mathrm{gm} \mathrm{BD}$. What are the rationale and precautions necessary with each of these drugs?

8. 1 litre of $\mathrm{N} /$ Saline with $40 \mathrm{mmols} \mathrm{KCl}$ over 8/24. Why Mr Aljunaibi requires potassium added to the ordered N/Saline?

9. You note that Mr Aljunaibi will be most likely discharged home on day 7 if his recovery is uneventful. What information do you think you will need to start this process?

\subsection{Program Participants}

The first program, conducted in November 2013, included 12 BN graduates (Group 1) who had previously completed a full GNI year and had failed the HAAD exam multiple times (mean $=5$, range 2-7) in the preceding 12 or 18 month period. This program was strongly remedial and focused on salvaging the professional futures of graduates facing premature conclusion of their nursing careers. The second program included $80 \mathrm{BN}$ students (Group 2); 18 had sat the HAAD exam at least once, and 62 had never sat the exam. This group included 2012 and 2013 graduates. The length of time since course completion presented a challenge, as scheduling issues meant some had waited 20 months for a place in the GNI program. Group 1 sat the exam in December 2013, one day after the review program.

\subsection{Program Evaluation}

Formal program evaluation was important to help evaluate key components of the success or any issues in the program. Evaluation was also noted as essential to inform future program planning. Thus a mixed method evaluation was designed which involved mid program focus groups and a formal end of program survey (Appendix 1). Ethical approval for the evaluation and the survey was granted by the Griffith University Institutional Research and Ethics Committee (IREC). Analysis of evaluation data provides previously unreported insights into the learning needs and experiences of locally trained graduate nurses preparing for a high-stakes nursing license exam in a Middle Eastern context. Egbert's framework of optimal learning conditions (Egbert, 2005) also proved to be useful for tool for the thematic analysis of qualitative comments and survey data.

\section{Results}

\subsection{Post Program Pass rates in the HAAD Licensure Exam}

Ten of the 12 Group 1 graduates (83\%) passed. Group 2 sat the exam in March 2014, also one day after the review program. Of the 79 students who were eligible to sit, 68 (86\%) passed. These results compare favorably with other external high-stakes examination benchmarks (National Council of State Boards of Nursing, 2013, 2014). The results for first-time sitters (Figure 1) are commensurate with American/Canadian National Council Licensure Exam (NCLEX) results, while pass rates for repeat sitters (Figure 2) were substantially better than those achieved in the American equivalents in 2013 and double in 2014. The fact that re-sitting participants performed equally as well as first time sitters suggests a problem of examination confidence and technique rather than a deficit in base knowledge. 


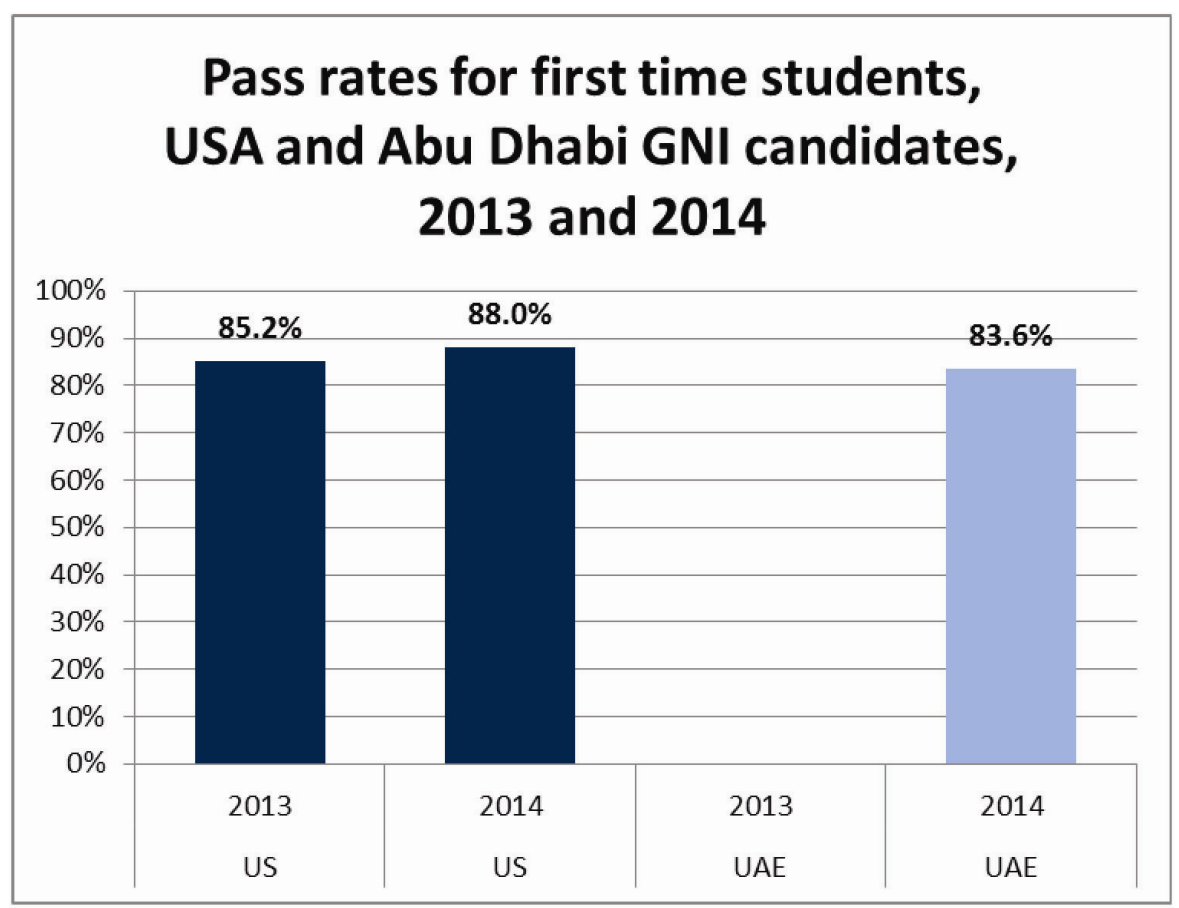

Figure 1. Pass Rates (first time attempts) for Abu Dhabi GNI Graduates vs USA/Canadian NCLEX Results

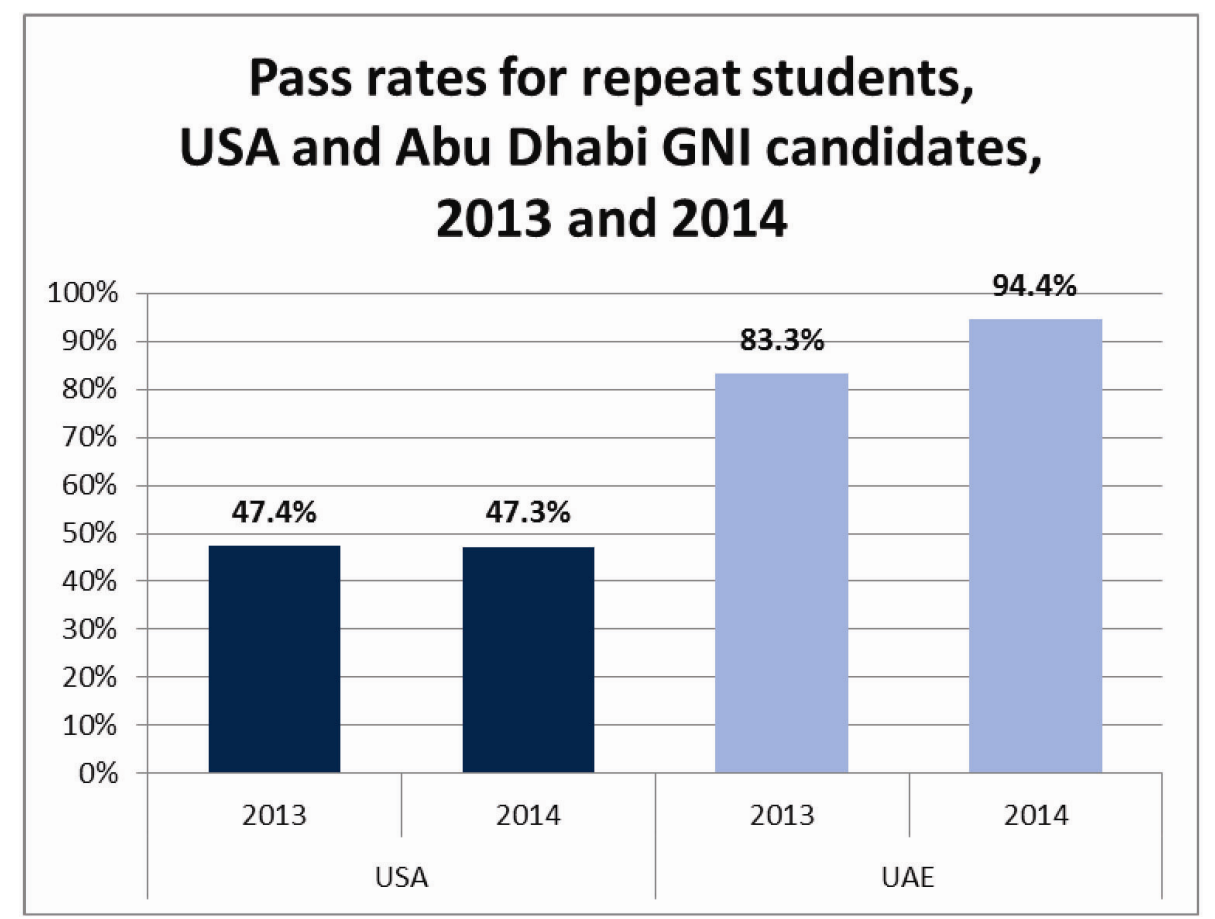

Figure 2. Pass Rates (repeat sitters) for Abu Dhabi GNI Graduates vs USA/Canadian NCLEX Results

Please note that the Group 1 (2013) cohort did not include any first time sitters

\subsection{Program Evaluation: Thematic Analysis \& Discussion}

The key measure of success of any educational initiative is the extent to which program objectives and the desired educational outcomes are achieved. Simply put, did it work? The pass results in the HAAD licensure exam signal success but these pass rates are not the only measure by which program success should be assessed. Equally important is the need for processes and measures to provide insight to student educational processes and learning 
experiences. Questions such as what was helpful and what was not so helpful to the learning process are important considerations to guide the program going forward. Thematic analysis using Egbert's framework proved to be a useful tool to guide analysis and discussion regarding the program and its associated learning outcomes.

4.2.1 Learner interaction: Target language, authentic audience

The program was planned and delivered through a partnership between English speaking university nursing faculty and local GNI coordinators. The university faculty and two GNI coordinators spoke English as their first language. One GNI coordinator was an Arabic speaker and able to provide Arabic language support when necessary - an important requirement for pastoral care and support. The use of English in the delivery of learning and assessment materials enabled commonly misunderstood terms to be identified and explained and strengthened language competence. The homogeneity of language required learners to listen, respond and interact with teaching faculty and each other in the language of the examination. This increased the authenticity of audience and the program. . Learners acknowledged a tendency to "revert to Arabic because it is easier" - participant 41 and "speak to each other in Arabic when we do not understand." - participant 76. However, the presence of fluent and sympathetic English speakers with a backup local Arabic speaking GNI coordinator was important for maintaining focus on the linguistic requirements for the HAAD exam.

\subsubsection{Development of critical thinking skills}

Focus on the nursing process and use of case studies is noted as an enabler in the development of critical thinking skills, nursing judgment, and in successful remediation and exam preparation programs (Colombraro, 1989; Frith et al., 2005; Hyland, 2012; Norton et al., 2006). Week one of the program included daily presentation and discussion of a clinical case study (Table 1). Learner perception of the usefulness of the case studies presented daily challenges for the teaching team. A further challenge was moving learners away from the preferred rote memory approach (Cowan, 2011; Dahl, 2011).

The teaching team remained firm in respect to the use of case studies as a method of teaching the nursing process and judgment in choosing the "best' answer for each question, resisting daily requests to "just give us the right answer"participant 54. The efficacy of this approach was confirmed through learner responses indicating that:

"To begin with we wonder about the use of case studies but at the end we really know how this helped to teach us about viewing the whole patient - we did better with the exams later cause of the case presentations because we thought about the whole patient and many things after learning more through the case presentations" - participant 9

"Case studies were very helpful as we saw so many things just in one case" - participant 36

Although learners acknowledged case studies were useful, they remained the least preferred learning activity, compared to short one-hour challenge tests or full three-hour exams (Figure $3 \& 4$ ).

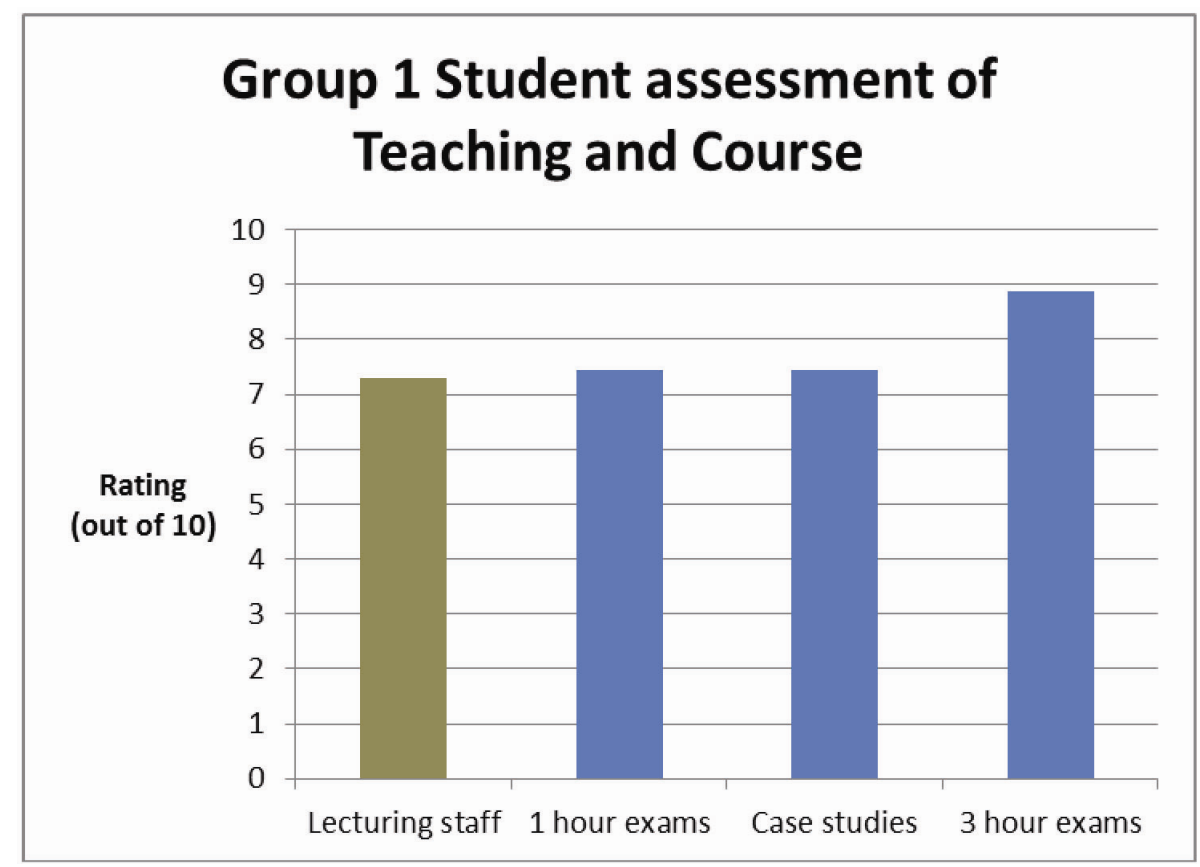

Figure 3. End of program assessment. Group 1 


\section{Group 2 Student assessment of Teaching and Course}

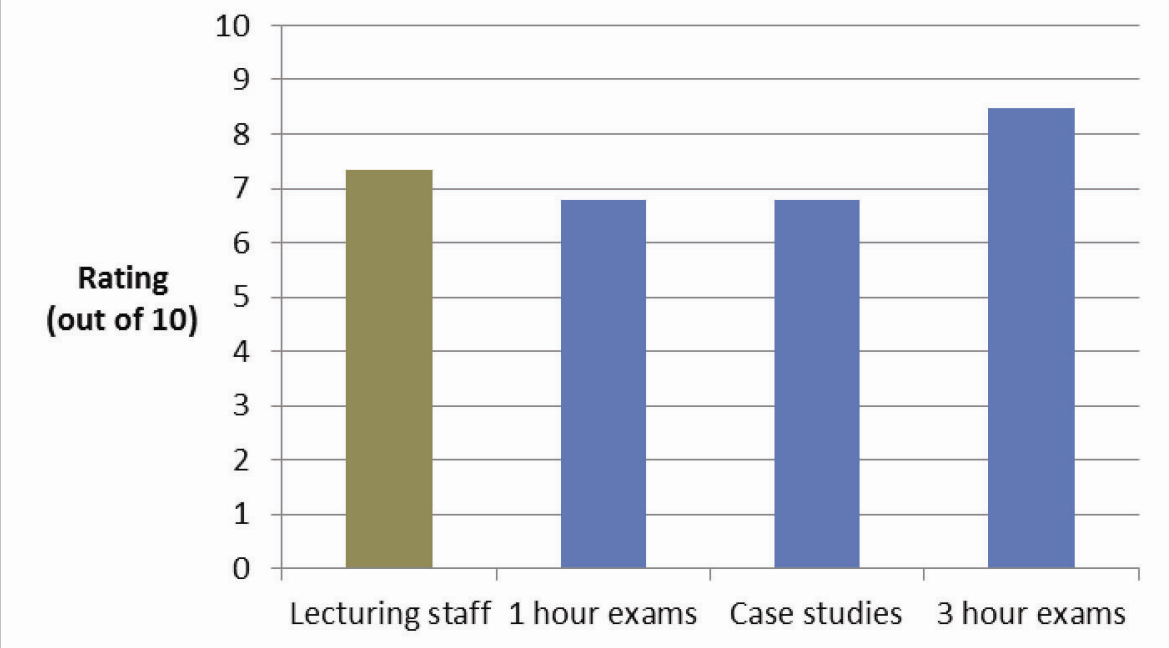

Figure 4. End of program assessment. Group 2

The push from some learners to revert to a systems and skills based approach persisted throughout the program, with some believing that they could pass the exam simply learning and memorizing content. Learner recommendations for additions to the program included:

"Focus on HAAD questions more"- participant 3

"Summarize the body system and disease instead of distributing case papers"-participant 7

"More treatments"- participant 69

"Practice more questions instead of case studies"- participant 54

"Divide into 3 weeks. Week 1 medical-surgical, Week 2 maternity and psychiatry and Week 3 General" 66

Learner feedback was considered in light of the relatively recent transition of Middle Eastern and UAE nursing education programs from skills-based diploma to competency-based degree qualifications. The teaching team concluded that focus on the nursing process and the inclusion of case studies should remain an important component of future programs.

Feedback about the learning context in which case studies were used included:

"But for the case study, it was not helpful because we are a big group. If we are a smaller group maybe it would be helpful" - participant 19

"The big groups are difficult"- participant 66

"Other students talking make it difficult to hear"- participant 79

This confirmed that the optimal environment for using case studies is in small groups with an acoustic environment that supports active listening and discussion.

\subsubsection{Authentic tasks}

The program included authentic learning activities directly relevant to successful high-stakes exam preparation. As noted, the first week of the program reviewed fundamental concepts, orientated students to the critical thinking requirements of the nursing process, and included short tests. From the outset, learners expressed preference for spending the majority of the program undertaking practice exams. Feedback indicated that:

"Doing exams over and over is useful - we are getting better with practice" - participant 11

"Having plenty of exam questions in different topics of nursing was extremely helpful"- participant 33

"3 hour exam most helpful" participants 5, 17, 36, 41, 43, 6, 76 
Practice exams and tests can make a significant difference to performance in final tests scores (Dunlosky, Rawson, Marsh, Nathan, \& Willingham, 2013; Walker et al., 2010). Learners felt that practice exams (with feedback) were a significant contributor to later success. A recurrent theme in the evaluation data is student recommendations that access to practice exams should be provided consistently throughout the undergraduate nursing program. Learners also reported that preparation for the HAAD exam should begin earlier in the undergraduate nursing program:

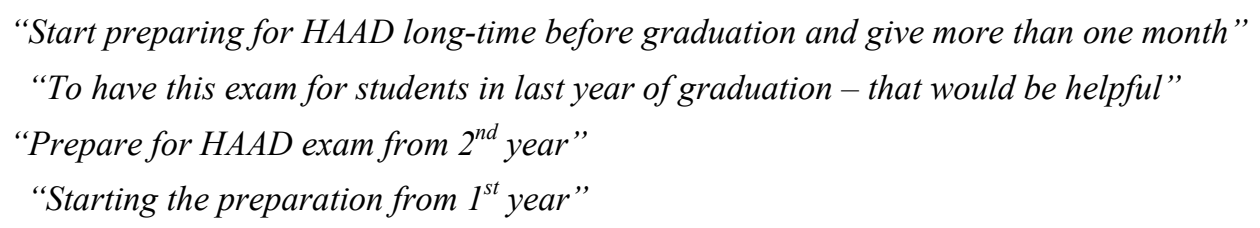

Learner feedback on this topic is an important consideration for ongoing delivery of undergraduate nursing programs in the UAE.

4.2.4 Program schedule and learner feedback

Learners received daily feedback on test and exam performance. Completed tests and exams were exchanged and marked by each learner's most closely located colleague. Results were recorded centrally allowing records to be maintained of daily performance, progress, and areas of strength or weakness. Assessments were returned to students, and group discussion addressed incorrectly answered questions. Learners were able to take assessment items home each day for further review and ongoing learning. Program evaluation data highlighted this was one of the most helpful learning strategies.

"Getting the answers and our results the same day was the best - we could take it home and correct all our mistakes and learn best"- participant 45

"Getting immediate feedback was the most helpful. Getting the answers so we know where we did wrong and could go back over it"- participant 32

"Discussion of the questions was very helpful"-participants 2, 5, 17, 41, 56, 58, 63, 79

No specific comments were received about time within the daily schedule. Positive responses regarding provision of feedback suggests that the program was balanced correctly. At the end of the program, most students indicated that they had enough practice testing and were exam ready. Others felt that some more time would be helpful.

"My recommendation is to extend two weeks as it will be more beneficial to expose to more information"participant 7

"Sitting our exam too early - it was difficult"- participant 68

The support from SEHA of paid study leave was a significant employer commitment. The achievement of results which are commensurate with global benchmarks in high-stakes nursing exams does not warrant extension of the program. However, review of exam timing would be appropriate. A two-day study break between the conclusion of the program and sitting the exam may provide an additional boost in learner confidence and exam readiness.

4.2.5 Learner autonomy

The program focused on teaching learners how to learn and develop autonomous learning skills. Answer sheets for assessment items were not provided despite repeated student requests. Instead, learners co-marked assessments and engaged in discussions about the best possible answer and how to search for answers. Teaching aids included a WIFI connected lap-top projected to a screen to show learners how to use library data-bases and Google to locate evidence-based information to help determine the best response. This technique was used to guide learners away from rote memorization of question and answer hand-outs. Learners resisted this deliberate move from a "Sage on the Stage"(King, 1993) p 30 teaching style towards constructivist pedagogy. In her book entitled Failure to Thrive in Constructivism: A Cross-Cultural Malady, (Dahl, 2011) Marilyn Dahl outlines factors associated with resistance to Western pedagogy in the Arab world and provides insights to equip Western teachers with skills for cross-cultural classrooms and transnational programs.

Many learners were annoyed by the constructivist approach which sought to actively engage them in the learning process, feeling that the teacher/s were inadequate and did not know the answers.

"We need to know what is in the HAAD exam, the facilitators were not able to give enough information about the HAAD exam"- participant 37

"Helpful is the program but not helpful is the teacher"- participant 54 
"If the tutor is experienced in the HAAD exam that would be helpful because the tutor does not know the real HAAD content"- participant 41

"First we were expecting that the tutors would be from HAAD itself so they could tell us what was in the exam ....."-participant 23

"In a scale of 1-10 I would give the training a 1-2 seriously because the tutors were not knowledgeable ... One of the tutors was all the time asking for us to search for answers on GOOGLE. Can you believe that, we are talking about evidence-based practice and he was telling us to GOOGLE and answer to us using GOOGLE"'- participant 26

The group who knew most about HAAD exam content was the cohort of 2013 graduates who had been provided with the opportunity to attend HAAD premises and undertake a mock exam in the final week of their program. In the face of student criticism, teaching faculty explained the role of the health regulator (HAAD), the educational providers, and the employer (SEHA). HAAD officials visited with the learners during the program and the strong partnership and cooperation between regulator, health service provider, and educational providers steered learners toward independent learning, critical thinking, and the ability to 'think like a nurse'.

Despite the successful outcome of pass-rates commensurate with long-established Western benchmarks, many students remained critical of the teaching faculty. For many, a teacher who does not provide hand-outs and speak to irrefutable facts is lacking in competence. This is a well-documented pedagogical challenge in the Arab world (Cowan, 2011; Dahl, 2011). However, while a number of learners remained critical (even though they passed the exam) others quickly adapted and eagerly engaged in the learning process.

"We got taught how to properly search for answers by ourselves and not just rely on the teachers"participant 36

"We have learned that every media counts - we know much better how to use on-line MCLEX, Google Scholar, and Wikipedia and can do this at the bus stop and anywhere we can be studying and increasing our knowledge. Every media counts and we can make every minute count" - participant 21

"The course helped in the gaps between our graduation and the exam - we think we now have a fair chance to pass" - participant 16

4.2.6 Remedial support and exam techniques

Provision of remedial support such as teaching study skills and exam techniques increased learner confidence and autonomy. To help assess support needs, learners who had previously failed the exam and were comfortable to share their experience were asked about the study techniques they had used. The most common technique involved memorizing NCLEX questions and answers and nursing program text books. Some had put their lives on hold for months, spending up to sixteen hours per day focused on rote memorization with little understanding of how to detect or determine a correct answer if the question changed slightly. Specific instruction in study skills and exam techniques yielded pass rates double those achieved by counterpart repeat sitters in the NCLEX exam in 2014 as stated earlier (Figure 2). It also increased the confidence level learners in both group 1 and 2 (Figure $5 \& 6$ ). 


\section{Group1 Confidence scores}

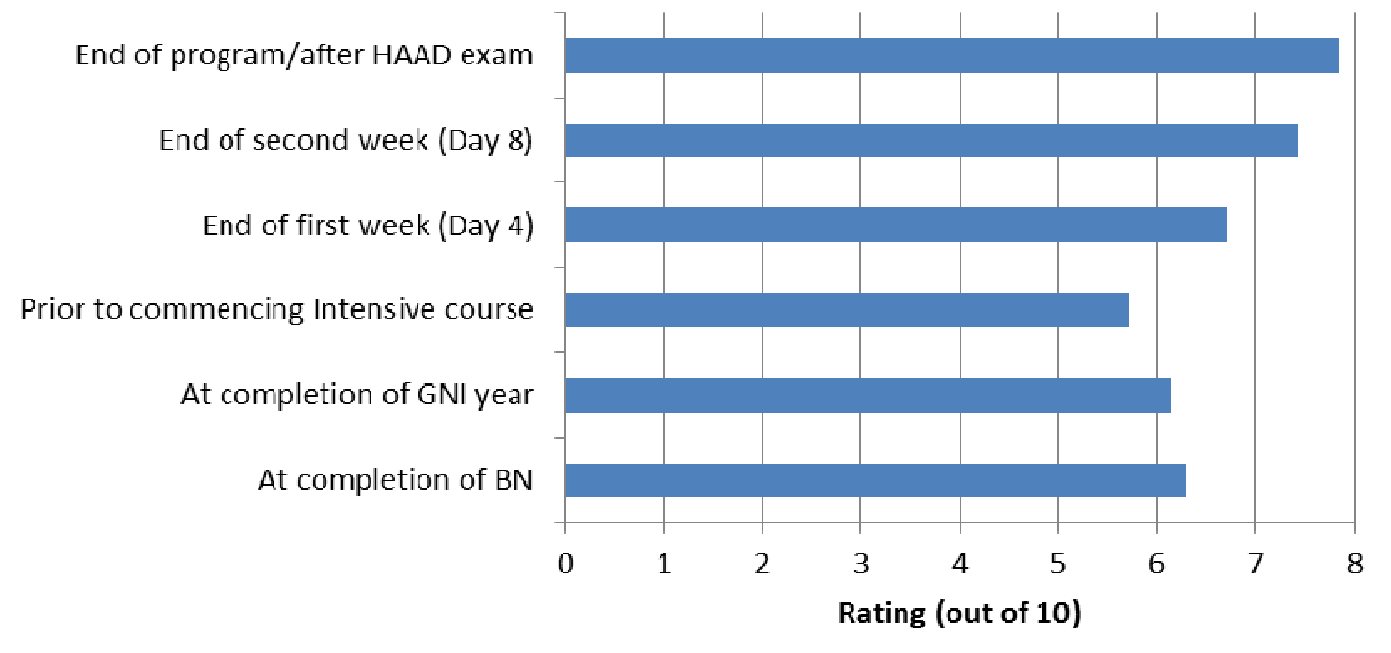

Figure 5. Group 1 confidence scores before, during, and after the program

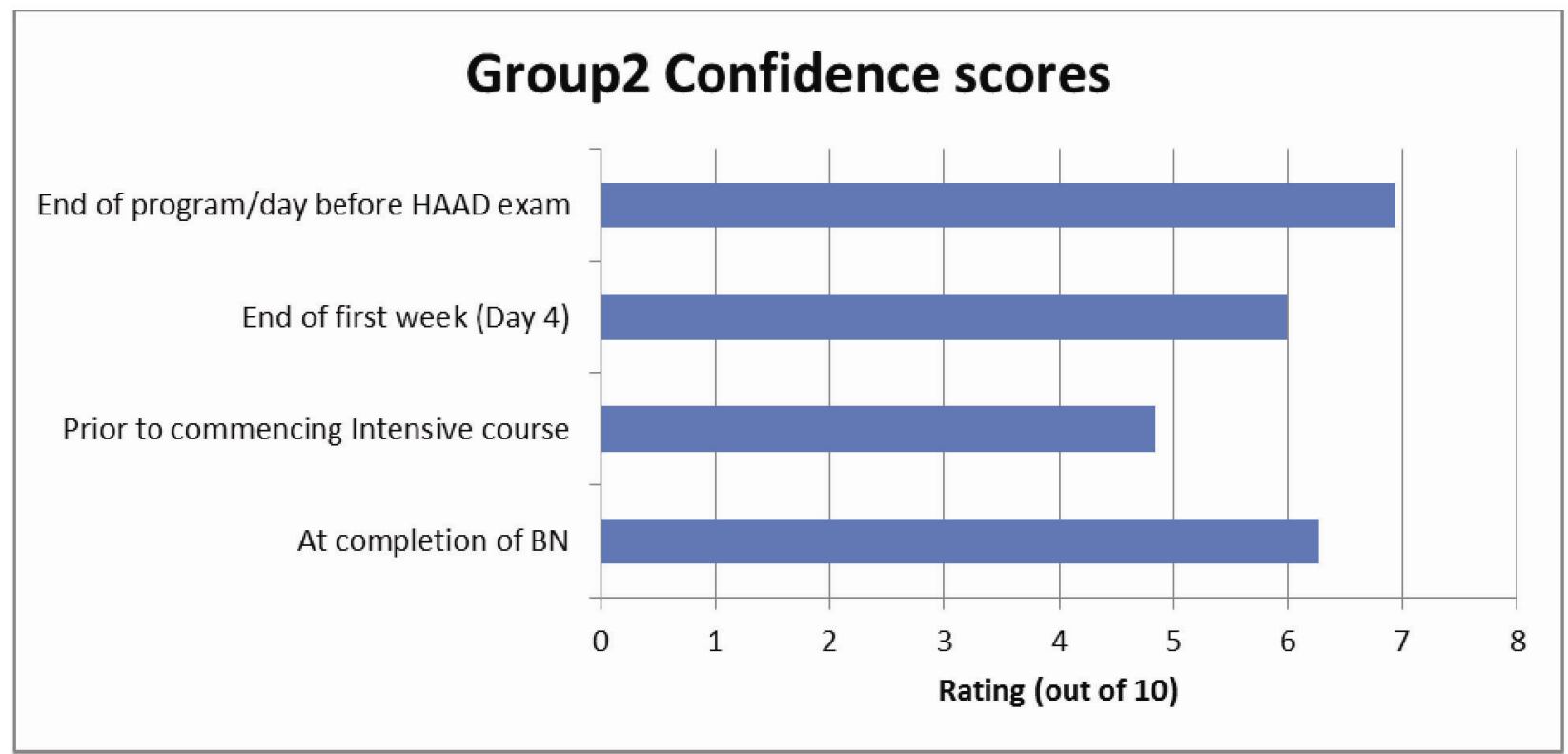

Figure 6. Group 2 confidence scores before, during, and after the program

"We learned how to improve our weaknesses"- participant 16

"Always read the question very careful and read it several times - watch out you understand the question properly"-participant 24

"We have learned to believe in ourselves"-participant 5, 9, 16, 22, 43, 65

"We learned how to time manage and how to sit for 3 hours and stay focused"- participant 77

4.2.7 Mindfully attending to the learning process

Dedicated sessions on the need for daily study, sufficient sleep, involvement in peer study groups, and broader family support helped engage graduates in the learning process and focus on the end goal of the examination. Active daily support including one-on-one pastoral care and counselling was provided by the Graduate Internship Coordinator in each SEHA facility. Students were encouraged to reflect on their study habits and daily routine.

"Eating of lunch and drinking properly is important otherwise we did not do so well in the tests"participant 22 
"We also learned to be responsible and bring all our stuff with us"- participant 74

"We learned it is important to sleep well before the exam to answer the questions right"-participant 75

"We study lots out of hours but learned never exceed 3-4 in a group study. Appoint a leader and a monitor to keep sharp focus and stop us talking"- participant 12

"Avoid group study in too big a groups - it does not work, there is too much talk. By yourself and group pairs are best for study"- participant 72

\subsubsection{Optimal stress/anxiety levels}

Maintaining a balance between assessment items and the optimal level of difficulty was a challenge, particularly given the breadth and diversity of learning needs across a large cohort of learners. Egbert encourages teaching faculty to create optimal stress levels by best matching the level of difficulty with learner skill (Egbert, 2005).

"Earlier exams were easy, need to be more difficult like last one - motivates you to do much better"participant 41

\subsubsection{Interacting and negotiating meaning}

An important aspect of program delivery was creating a culture of collegiality, sharing, and fun. During the program, learners developed a sense of pride in their professional identity as graduate nurses, with many personal and professional friendships developed and strengthened.

"Community of nurses - it has built strong groups and friendships between graduates and all of us do strengthen our nursing"- participant 44

“We feel a team"- participant 9, 5, 32, 45, 54, 63, 77

\section{Conclusion and Recommendations}

The costs of failure in high-stakes nurse license exams are significant for health services, educational providers, and individual graduates (Roa, Shipman, Hooten, \& Carter, 2011). The successful teaching and learning initiative outlined in this case study provides valuable insights into cultural and contextual issues in transnational program delivery. This educational initiative highlights how teaching and assessment strategies can be designed to support nursing graduates to be successful in the high-stakes HAAD examination in the Emirate of Abu Dhabi. The following recommendations are made in respect to ongoing delivery of the program.

Future exam preparation programs: Further programs will be an important enabler of successful transition to practice for nursing graduates in Abu Dhabi and should be offered for each graduating class

Pedagogy: A continued focus should be maintained on teaching and assessment strategies which actively engage learners in the learning process and develop the critical thinking skills to 'think like a nurse.'

Learning preferences of Arab students: Further research is needed to better understand and support the learning preferences and styles of Arab learners transitioning to autonomous health professional roles.

The Bachelor of Nursing program: Constructivist pedagogy and early focus on the attainment of nursing licensure should be integrated into the delivery of the undergraduate nursing program, with consideration given to sitting the exam soon after graduation.

The HAAD license exam: The provision of more information, including publically available access to samples of the HAAD nurse license exam is recommended as an important part of the ongoing development of systems for the education and regulation of the UAE's nursing workforce.

While the effectiveness of individual strategies or the extent to which the success of the combined strategies are replicable or generalizable are not yet known, it is clear that the "the more educators know about what will make students successful on the licensure exam, the better the chance that interventions will be successful" (Zweighaft, 2011). On this basis, continued delivery and evaluation of this program along with research in best practices in transnational nursing programs and Arab speaking contexts is recommended.

\section{Acknowledgement}

The authors acknowledge the research assistance provided by Audrey Holmes who undertook grammatical reviews and assisted with the management of Endnote functions and formatting requirements within this manuscript. Acknowledgement is also given to the Griffith University School of Nursing and Midwifery as the source of the case study example profiled in this manuscript. 


\section{References}

al Mahmud, A. (2013). Constructivism and Reflectivism as the Logical Counterparts in TESOL: Learning Theory versus Teaching Methodology TEFLIN Journal, 24(2), 237-257.

Atkinson, D. (1999). TESOL and Culture. TESOL Quarterly, 33(4), 625-654. http://dx.doi.org/10.2307/3587880

Burton, M. (2011). Revisioning Transition-to-Practice Issues. Kentucky Nurse, 59(1), 1.

Charlesworth, Z. M. (2008). Learning styles across cultures: suggestions for educators. Education + Training, 50(2), 115-127. http://dx.doi.org/10.1108/00400910810862100

Colombraro, G. C. (1989). NCLEX-RN Preparation: Enabling Candidates to Pass. The Journal of Continuing Education in Nursing, 20(6), 261-267.

Cowan, J. (2011). Teaching and Learning in the Arab World (C. Gitsaki Ed.). USA: Peter Lang.

Crow, C. S., Handley, M., Shaw-Morrison, R., \& M., S. (2004). Requirements and interventions used by BSN programs to promote and predict NCLEX-RN success: A national study. Journal of Professional Nursing, 20(3), 174-186. http://dx.doi.org/10.1016/j.profnurs.2004.04.004

Culleiton, A. L. (2009). Remediation: A closer look in an educational context. Teaching and Learning in Nursing, 4(1), 22-27. http://dx.doi.org/10.1016/j.teln.2008.07.001

Dahl, M. (2011). Failure to thrive in constructivism a cross-cultural malady. McGill University, Canada: Sense Publishers.

Dunlosky, J., Rawson, K. A., Marsh, E. J., Nathan, M. J., \& Willingham, D. T. (2013). Improving Students' Learning With Effective Learning Techniques: Promising Directions From Cognitive and Educational Psychology. Psychological Science in the Public Interest, 14(1), 4-58. http://dx.doi.org/10.1177/1529100612453266

Dyess, S. M., \& Sherman, R. O. (2009). The First Year of Practice: New Graduate Nurses' Transition and Learning Needs. The Journal of Continuing Education in Nursing, 40(9), 403-410. http://dx.doi.org/10.1177/1529100612453266

Egbert, J. (2005). Introduction: Principles of CALL J. Egbert (Ed.) CALL Essentials: Principles \& Practice in CALL Classrooms (pp. 1-206).

Egbert, J., Hanson-Smith, E., \& Chao, C. (2007). Foundations of Teaching \& Learning. In J. Egbert \& E. Hanson-Smith (Eds.), CALL Environments: Research, Practice \& Critical Issues. Alexandria, VA: TESOL.

El-Haddad, M. (2006). Nursing in the United Arab Emirates: an historical background. International Nursing Review, 53(4), 284-289. http://dx.doi.org/10.1111/j.1466-7657.2006.00497.x

Frith, K. H., Sewel, J. P., \& Clarke, D. J. (2005). Best Practices in NCLEX-RN Readiness Preparation for Baccelaureate Student Success. CIN: Computers, Informatics, Nursing, 23(6), 322-329.

Hannah, V. M. (2006). The big picture: learning to think like a nurse. Journal of Nursing Education, 45(6), $239-240$.

Hannawi, S., \& Al-Salmi, I. (2013). Health workforce in the United Arab Emirates: analytic point of view. The International Journal of Health Planning and Management, 1-10. http://dx.doi.org/10.1002/hpm.2198

Heffernan, T., Morrison, M., Basu, P., \& Sweeney, A. (2010). Cultural differences, learning styles and transnational education. Journal of Higher Education Policy and Management, 32(1), 27-39. http://dx.doi.org/10.1080/13600800903440535

Hillege, S. P., Catterall, J., Beale, B. L., \& Stewart, L. (2014). Discipline matters: Embedding academic literacies into an undergraduate nursing program. Nurse Education in Practice, 14(6), 686-691. http://dx.doi.org/10.1016/j.nepr.2014.09.005

Hyland, J. R. (2012). Building on the evidence: Interventions promoting NCLEX success. Open Journal of Nursing, 2(3), 231-238. http://dx.doi.org/10.4236/ojn.2012.23036

Johnson, A. N. (2009). NCLEX-RN Success with Boot Camp. Nursing Education Perspectives, 30(5), $328-329$.

Kaufman, J. S., Baradell, J., Calhoon, M., \& Durham, C. (1987). Preparation for NCLEX-RN: An Innovative Course. Journal of Nursing Education, 26(6), 250-253. 
King, A. (1993). From Sage on the Stage to Guide on the Side. College Teaching, 41(1), 30-35. http://dx.doi.org/10.2307/27558571

Kirk, D. (2010). The Development of Higher Education in the United Arab Emirates. The Emirates Occasional Papers, 74, 1-51.

Latham \& Watkins. (2013). 2013 Update: Healthcare Regulation in the United Arab Emirates Client Alert White Paper: Latham \& Watkins.

McManus, I. C., \& Ludka, K. (2012). Re-sitting a high-stakes postgraduate medical examination on multiple occassions: non-linear multilevel modelling of performance in the MRCP (UK) examinations. BMC Medicine, 10(60), 1-19. http://dx.doi.org/10.1186/1741-7015-10-60

Morton, A. M. (2004). Improving NCLEX Scores With Structured Learning Assistance. Nurse Educator, 31(4), 163-165. http://dx.doi.org/10.1097/00006223-200607000-00009

National Council of State Boards of Nursing. (2013). NCLEX Table of Passrates 2013 (pp. 1). https://www.ncsbn.org/1237.htm: National Council of State Boards of Nursing.

National Council of State Boards of Nursing. (2014). NCLEX Table of Passrates 2014 (pp. 1). https://www.ncsbn.org/1237.htm: National Council of State Boards of Nursing.

Neito, S. (2007). Affirming Diversity: The Socio-political Context of Multicultural Education (4th p/back ed.). Bonston, USA: Allyn \& Bacon.

Norton, C. K., Relf, M. V., Cox, C. W., Farley, J., Lachat, M., Tucker, M., \& Murray, J. (2006). Ensuring NCLEX-RN Success for First-Time Test-Takers. Journal of Professional Nursing, 22(5), 322-326. http://dx.doi.org/10.1016/j.profnurs.2005.11.004

Pennington, T. D., \& Spurlock, D. (2010). A Systematic Review of the Effectiveness of Remediation Interventions to Improve NCLEX-RN Pass Rates. Journal of Nursing Education, 49(9), 485-492. http://dx.doi.org/10.3928/01484834-20100630-05

Roa, M., Shipman, D., Hooten, J., \& Carter, M. (2011). The costs of NCLEX-RN failure. Nurse Education Today, 31(4), 373-377. http://dx.doi.org/10.1016/j.nedt.2010.07.009

Sagar, P. L. (2014). Transcultural nursing education strategies. New York, NY: Springer Publishing Company.

Simon, E. B., McGinniss, S. P., \& Krauss, B. J. (2013). Predictor Variables for NCLEX-RN Readiness Exam Performance. Nursing Education Perspectives, 34(1), 18-24. http://dx.doi.org/10.5480/1536-5026-34.1.18

TESOL International Association. (2002). Standards for Adult Education TSL Programs (pp. 1-219): Teachers of English to Speakers of other Languages Inc.

Twort, C., \& Mucklow, J. (2011). How to write high-quality questions for a high-stakes examination. Clinical Medicine, 11(3), 227-230. http://dx.doi.org/10.7861/clinmedicine.11-3-227

Walker, J. D., Brooks, D., K., H., Fall, J. A., Peiffer, R. W., Schnell, R., \& Schottel, J. L. (2010). Practice Makes Perfect? Assessing the Effectiveness of Online Practice Exams in Learning Biology Concepts Retrieved August 10, 2014, from https://it.umn.edu/prod/groups/oit/@pub/@oit/@web/@evaluationresearch/documents/article/oit_article_24770 5.pdf

Zweighaft, E. L. (2011). National Standardize Speciality Exams Improve Predictive Exit Exam Scores and NCLEX-RN Success. Paper presented at the 41st Bienniel Convention, Grapevine, Texas, USA. 


\section{[Appendix 1]}

Dear Graduate,

Thank you for attending the Griffith University/Fatima College HAAD examination preparation classes. You participation was much appreciated.

The following questionnaire has been designed to find out more about you and your colleagues and your perceptions of the learning experience. Your answers are completely private and confidential and will be de-identified and aggregated for publication purposes. If you have any questions please do not hesitate email xxxx for assistance or advice.

1. In which year were you born? Year:

2. What is your Gender: Male/ Female: Answer:

3. In what country were you born?

4. How long have you lived in the UAE?

5. What was your first fluent language?

6. What other languages do you speak?

7. How old were you when you mastered the English language fluently?

8. In which year and month did you commence the Bachelor of Nursing Program?

Year: Month:

9. In which year and month did you complete the Bachelor of Nursing Program?

Year: Month:

10. Prior to the commencement of the Griffith University/Fatima College HAAD examination preparation on how many occasions had you attempted the licensure exam with HAAD? No:

11. On a scale of 1 to 10 , where $1=$ Not confident at all and $10=$ Extremely confident how confident were you that you would have enough knowledge and information to pass the HAAD at the following points in time.

1. At completion of your Bachelor of Nursing program? Scale No:

2. Prior to commencing the GU/FCHS intensive course? Scale No:

3. At the end of the first week of the GU/FCHS intensive course? Scale No:

4. At the end of the second week of the GU/FCHS intensive course? Scale No:

5. Immediately prior to commencing the HAAD exam? Scale No:

12. On a scale of 1 to 10 , where $1=$ Not helpful at all and $10=$ Extremely helpful how helpful do you believe the following activities were to prepare you for the HAAD exam?:

1. The skills and abilities of the facilitators? Scale No:

2. The short 1-hour examinations used in the first week? Scale No:

3. The short case studies used in the first week? Scale No:

4. The longer 3-hour examinations used in the second week? Scale No:

5. Group tuition time to review and discuss answers to examination questions? Scale No:

6. What other elements of the preparation program do you believe where helpful, why?

7. What other elements of the preparation program do you believe where NOT helpful, why?

13. What other advice would you give to future students and lecturers to help students prepare for the HAAD exam?

14. What other advice would you give HAAD examiners about the exam processes? 